\title{
The Olfactory System: An Emerging Model for Studying Cilipoathies.
}

\author{
D.J. Rodriguez-Gil. \\ Yale University School of Medicine, Department of Neurosurgery, New Haven, CT 06510
}

The olfactory epithelium is located in the most dorsal region of the nasal cavity and contains three types of cells: olfactory sensory neurons (OSNs, responsible for odorant detection), sustentacular cells (which give support), and basal stem cells (that regenerate the other two populations) [1,2]. OSNs are bipolar cells with a single dendrite extending to the nasal cavity and a single axon to the underlying lamina propria that extends and synapses in the olfactory bulb [3]. The apical dendrite ends in a knob that contains multiple basal bodies, from which non-motile cilia (10-30) extend in the surface of the epithelium [4]. These cilia lie in the surface of the epithelium and increase the surface for odorant reception approximately 40 times [5]. Mammalian olfactory cilia can be as long as $60 \mu \mathrm{m}$ [5], and even though OSN cilia ultrastructure presents a classical $(9+2)$ microtubule organization, the absence of dynein arms makes them immotile [6].

Ciliary function in OSNs has been well described because of its relevance in odor perception and because is where sensory transduction occurs. Olfactory receptors were discovered in 1991 [7], are GPCRs and, in mice there are as many as $\sim 1100$ genes coding for odorant receptors [8]. Interestingly, each OSN seems to express only one odorant receptor [9]. Although odorant receptors were observed throughout the entire OSN, of relevance for this symposium is the notion that cilia harbor the seven transmembrane odorant receptors and all the components of the transduction cascade. Upon activation by a specific odor, the odorant receptor activates a stimulatory protein $\mathrm{G}$, which activates adenylyl cyclase III increasing cAMP concentration. This increase in cAMP opens cyclic nucleotide gated channels and the depolarization of the OSN occurs [3]. All these proteins have been identified, cloned, characterized and many of them knocked-out and proven important for the transduction signaling. Despite how much we know on the odorant transduction signaling mechanism in OSN cilia, little is known about the transport mechanisms controlling trafficking in and out of the OSN cilia.

Mutations in ciliary or basal body proteins, both in humans and animal models, showed a plethora of clinical features, being some of the most common ones retinitis pigmentosa, renal cystic disease, polydactyly, situs inversus, and mental retardation. Different syndromes such as Bardet-Biedl (BBS), Senior-Loken, Joulbert (JS) or Meckel-Gruber (MKS) have been classified based on the spectrum of clinical features manifested. Many of the proteins characterized as being involved in some of the previously mentioned syndromes, have also been recently shown to be expressed in the olfactory system. Nonetheless, linking both, ciliary deficiencies and olfactory deficits has not been obvious up to the last few years [10-13]. Loss of function of BBS-1 and BBS-4 lead to structural and functional deficit in mice models [10] and to a decreased olfactory function both in human and mice presenting a BBS-4 deletion [10,11]. Moreover, CEP290 is a ciliary/centrosomal protein that has been recently shown to alter olfactory perception [12]. Both human patients and mice with a mutation in CEP290 exhibited severe abnormal olfactory function (humans) or anosmia (mice).

We have been interested in the expression pattern and role of other ciliary proteins known to cause ciliopathies. We analyzed the expression of polycystin-1 (PC-1), polycystin-2 (PC-2), MKS-1 and MKS-3 in the olfactory system in wild-type mice using RT-PCR and immunohistochemistry. PC-1 and PC-2 are transmembrane proteins suggested to be involved in multiple signaling pathways [14]. MKS-1 is a cytoplasmic protein associated with the base of the cilium, and MKS-3 is a predicted transmembrane protein found in the cilia [15]. We characterized the alterations observed in the olfactory system in a conditionally knocked-out PC-1 mouse model, in a loss of function MKS-1 mouse model [16] and in a loss of function MKS-3 rat model [17]. Detailed analysis of the olfactory epithelium with confocal and electron microscopy showed a variety of phenotypes including disorganized olfactory epithelium, and altered ciliary ultrastructure 
and number. Taken together these results improve our knowledge on the role of these proteins and increase the relevance of the olfactory system as a suitable model to study ciliopathies [18].

\section{References}

[1] Cuschieri et al., J. Anat. 119 (2) (1975a) 277.

[2] Cuschieri et al., J. Anat. 119(2) (1975b) 471.

[3] S. Firtesein, Nature. 413 (2001) 211.

[4] B.P.M. Menco, Chem Senses. 22 (1997) 295.

[5] B.P.M. Menco, Microsc Res Tech. 22 (1992) 215.

[6] B.P.M. Menco, Cell Tissue Res. 235 (1984) 225.

[7] L. Buck and R. Axel, Cell. 65 (1991) 157.

[8] X. Zhang, Proc. Natl Acad. Sci. USA. 101 (2004) 14168.

[9] P. Mombaerts, Curr. Opin. Neurobiol. 14 (2004) 31.

[10] H.M. Kulaga, Nat. Genet. 36 (2004) 994.

[11] A. Iannaccone et al., Am. J. Med. Genet. 132 (2005) 343.

[12] D.P. McEwen et al., Proc Natl Acad Sci USA. 104 (2007) 15917.

[13] P.M. Jenkins et al., J Neurosci. 29(34) (2009) 10541.

[14] H. Chapin and M.J. Caplan, JCB. 191 (2010) 701.

[15] C.V. Logan, Mol Neurobiol. 43 (2011) 12.

[16] S.D. Weatherbee et al., Hum. Mol. Genet. 18(23) (2009) 4565.

[17] V.H. Gattone et al., Anat Rec A Discov Mol Cell Evol Biol. 277 (2004) 384.

[18] The author acknowledges the contribution of all the collaborators on this project and the support of

$\mathrm{NIH}$, funding multiple members of this project. 